\title{
Paisaje lingüístico urbano y rural: parámetros de caracterización
}

\author{
Urban and rural linguistic landscape: characterization parameters
}

CARMEN FERNÁNDEZ JUNCAL

UNIVERSIDAD DE SALAMANCA

Artículo recibido el / Article received: 2018-10-29

Artículo aceptado el / Article accepted: 2019-01-26

RESUMEN: El presente estudio tiene como objetivo determinar el peso de diferentes parámetros en la descripción del paisaje lingüístico de dos zonas de diferente ámbito: urbano y rural. Partimos de las investigaciones llevadas a cabo en dos demarcaciones próximas de la variedad centro-norte peninsular del español en la frontera con el vasco. Aunque se trata de áreas que, por razones geográficas, administrativas y políticas presentan entre sí disparidades en su perfil lingüístico, la clasificación cuantitativa de sus señales lingüísticas nos permite establecer patrones diferenciados para los dos entornos mencionados.

La influencia de la estructura económica en el espacio público de cada entorno, rural o urbano, se observa en el paisaje lingüístico de ambos: por una parte, comparten la misma vitalidad en su renovación. Por otra parte, se detectan diferencias si consideramos otros criterios: el paisaje rural, que ha sido poco estudiado, es más institucionalizado, más dependiente, por lo tanto, del impulso público, subsidiario de autores y referentes externos a la comunidad, limitado en sus funciones y representaciones sociales.

Palabras clave: paisaje lingüístico, rural, urbano, parámetros sociales, representación social

ABSTRACT: The aim of this paper is to determine the weight of different parameters in the description of the linguistic landscape of two zones of different scope: urban and rural. The starting point was a previous investigation carried out in two nearby sites, in the limits between the North-Central peninsular variety of the Spanish and the Basque. Although they are areas that, for geographical, administrative and political reasons have a disparate linguistic profile, the quantitative classification of their linguistic signals allows us to establish patterns for the two mentioned environments.

The influence of the economic structure in the public space, rural or urban, can also be observed in the linguistic landscape: on the one hand, they share the same vitality in its renewal. On the other hand, differences can be detected if we consider other criteria: the rural landscape, which has been little studied, is more 
institutionalized, more dependent, therefore, on public initiative, more subsidiary to authors and references external to the community, more limited in its functions and social representations.

Keywords: Linguistic Landscape, Rural, Urban, Social Parameters, Social Representation

\section{EL PAISAJE LINGÜÍSTICO Y EL MEDIO RURAL}

Como nos recuerda Gorter (2006), el término paisaje remite en las diferentes lenguas a dos dimensiones de observación: una más técnica y objetiva frente a otra más emocional y subjetiva. Este doble enfoque se traslada a la perspectiva de análisis del paisaje lingüístico (también PL): una conceptual o de contenidos y otra más simbólica o de identidades, que permite, de acuerdo con los postulados iniciales de Landry y Bourhis (1997), medir la diversidad de lenguas y la vitalidad etnolingüística de cada una de ellas dentro del territorio. Antes de que estos autores fijaran las bases de la subdisciplina, la presencia del lenguaje en el espacio público había sido objeto de atención de otros estudiosos (Spolsky, 2009), pero 1997 marca una fecha de implosión de estas investigaciones. Desde entonces los estudios se han sucedido tanto en áreas clasificadas como bilingües como en ámbitos de convivencia multilingüe por efecto de los movimientos poblacionales que se han venido produciendo de manera especialmente intensa en las últimas décadas. Los países monolingües han constituido siempre una auténtica excepción en nuestro mundo, pero además la globalización ha acelerado los flujos migratorios y la difusión de productos culturales (Gorter, 2006; y Cenoz y Gorter, 2008). Así pues, el valor de estas investigaciones es plasmar la realidad lingüística de las comunidades donde se encuentran (Franco-Rodríguez, 2008) y también las actitudes que esas lenguas generan (Blackwood, 2015; Pavlenko, 2010), dar cuenta de la Gestalt que constituye el conjunto de elementos dispares que componen el PL (Ben-Rafael y BenRafael, 2015; y Guerra Salas, 2018).

La trayectoria de estos estudios (Huebner, 2016) ha ido en paralelo al afianzamiento de los fundamentos metodológicos (Shohamy y Gorter, 2009), una delimitación de las áreas temáticas (Barni y Bagna, 2015), la creación de taxonomías para el objeto de estudio (Muñoz Carrobles, 2010) y la fijación de los parámetros de análisis de los corpus creados, que deben considerar factores de influencia más allá de lo lingüístico. La perspectiva ha de ser inevitablemente multidisciplinar: por su propia idiosincrasia, la descripción del paisaje lingüístico debe emplear fundamentos y herramientas de otras ciencias: la sociología, la geografía urbana, la psicología perceptiva, la etnografía o la antropología. Quedan retos por afrontar, como abrir camino a otras dimensiones comunicativas del paisaje (lo oral, lo icónico) (López Serena, 2008; Gorter, 2012), establecer procedimientos que permitan la comparación diatópica y diacrónica (Blackwood, 2015) o determinar el papel que desempeñan las actitudes en el origen y transformación de los signos. ${ }^{1}$

Apenas contamos con investigaciones fuera del medio urbano como las de Juffermans \& Coppolse (2012) en Gambia, Pons (2014) en la frontera hispano-portuguesa o Fernández Juncal (en prensa) en Cantabria-Vizcaya. Esto resulta lógico ya que, por su

\footnotetext{
${ }^{1}$ Utilizaremos indistintamente los vocablos signos (Backhaus, 2006) y textos (Franco-Rodríguez, 2008) para designar las unidades que constituyen el corpus.
} 
propia definición, el PL concentra su atención en las ciudades. ${ }^{2}$ Estas se caracterizan por su diversidad interna, por la estratificación poblacional, lo que repercute directamente en la pluralidad de actividades llevadas a cabo por agentes que actúan de manera autónoma $y$, por lo tanto, no obedecen a un plan establecido (Burr, 2003). Se trata de un lugar de atracción para las personas y para la actividad económica (Barni y Bagna, 2015), el foco donde se crean redes globales interconectadas de signos comunes y, al tiempo, diferenciales (Blommaert, 2010). No obstante, en palabras de Gorter (2006: 86),

In the countryside and in natural areas which are largely uninhabited, there are no, or only a very small number of, signs. In our world today there is little pure nature in a literal sense left because almost every spot has been 'touched' by human beings and traces of their presence have been left behind and with it linguistic tokens.

El trabajo que presentamos rompe con el principio casi general del carácter urbano de los estudios de PL buscando los rasgos que definen el exiguo pero existente paisaje lingüístico rural, que presenta características propias. Antes de detallarlas conviene repasar brevemente los rasgos socioeconómicos más destacados (Segrelles Serrano, 2011) que definen el medio rural ${ }^{3}$ porque, sin duda, tendrán consecuencias en su PL: baja densidad de población, debida a una economía basada en la explotación de los recursos propios, separación borrosa entre población activa e inactiva, niveles de renta inferiores a los de la población urbana y acceso algo más restringido a las infraestructuras y servicios. Como vemos, muchas veces el medio rural no se define por sí mismo sino en contraposición a lo urbano. En la actualidad la globalización ha conducido a la homogeneización y terciarización de las formas de vida también en esa esfera, lo que ha provocado, bien por evolución natural bien por el fenómeno de la neorruralización, la incorporación de nuevos servicios, nuevas tecnologías y nuevas técnicas (Baigorri, 1995).

Como comprobaremos, el ámbito rural presenta su propio PL, con más limitación de agentes, menos presencia comercial y, por lo tanto, con más influjo de la intervención pública. En contrapartida, algunos conceptos o técnicas con relevancia en medio urbano (prominencia, método de la valoración económica, etc.) son difícilmente aplicables a este entorno.

\section{CONTEXTO DE LA INVESTIGACIÓN}

Este estudio se centra en dos áreas geográficas próximas, pero de diferente carácter. ${ }^{4}$ Por una parte, tenemos el centro urbano de Castro Urdiales, municipio de la comunidad de Cantabria muy próximo a Bilbao. De hecho, se ha definido como un núcleo perimetropolitano que pertenece al área funcional de Bilbao (Delgado Viñas, 2018), aunque nunca se ha incluido como tal por pertenecer administrativamente a una comunidad autónoma diferente. La ciudad de Castro, como demuestran las cifras demográficas, es de carácter urbano. Oficialmente es el tercer municipio de Cantabria en número de habitantes, 32144 habitantes (INE, 2014). Sin embargo, considerando la población de hecho (población flotante que no está empadronada en el municipio pero

\footnotetext{
2 De hecho, Spolsky (2009) y Gorter (2013: 191) prefieren la denominación linguistic cityscape a la de linguistic landscape.

${ }^{3}$ El límite mínimo oficial (Ministerio de Fomento) y generalmente aceptado para que un municipio sea considerado urbano es de 10000 habitantes.

${ }^{4}$ Este trabajo tiene como punto de partida una investigación previa en las zonas señaladas que indagaba acerca de la influencia de la política lingüística en la presencia del vasco en el PL de las citadas localidades.
} 
reside en él regularmente), los datos no oficiales ${ }^{5}$ doblan esa cifra (casi 70000 ), de forma que más de la mitad de los habitantes de la población no figura como tal sino que está censada en la comunidad autónoma vecina. El número de habitantes se mantuvo estable entre 1900 y 1990, pero experimentó un incremento muy notable, casi el doble, a partir de $1996 .{ }^{6}$ Por otra parte, la estructura económica se concentra sin lugar a dudas en el sector terciario, ${ }^{7}$ con un gran peso de la industria turística, que ha hecho experimentar un gran desarrollo del comercio y la hostelería.

Por otra parte, disponemos de los datos correspondientes a las localidades de La Matanza, capital del municipio de Valle de Villaverde, en Cantabria, ${ }^{8}$ y Trucíos/Turtzioz, la capital del municipio del mismo nombre, en la provincia de Vizcaya. Ambas difieren en su situación administrativa, política y lingüística, pero, lo que nos importa para este caso, tienen en común ser colindantes y ser indiscutiblemente núcleos rurales. En 2017 el Valle de Villaverde contaba con 306 habitantes (densidad de 15,67) y Trucíos-Turtzioz con 510 (densidad de 16,39). Estos datos confirman el descenso de población: en TrucíosTurtzioz se ha pasado de una población de 865 habitantes en 1800 a los actuales 510 pasando por los 1038 de 1920. Lo mismo ha ocurrido en Valle de Villaverde, que contaba con 678 habitantes a mediados del siglo XIX y llegó a tener 764 en 1940, más del doble que en la actualidad. Se trata, por consiguiente, de dos unidades administrativas de carácter marcadamente rural, como señalan las propias cifras demográficas y la dispersión de población. La evolución demográfica ha sufrido diversos movimientos producto de la baja natalidad y el éxodo rural. La economía también ha pasado de depender exclusivamente del sector primario a diversificarse hacia el sector terciario, lo cual, como veremos, tiene consecuencias en su PL.

Se trata de demarcaciones geográficas que presentan un perfil lingüístico distinto. A pesar de que todas comparten ser calificadas históricamente como castellanohablantes, la influencia de la política lingüística del Gobierno Vasco a favor del euskera hace que para el caso de Trucíos/Turtzioz se empiecen a mostrar los efectos del proceso de normalización del euskera en el aumento de neohablantes y también en su paisaje lingüístico. Valle de Villaverde, municipio colindante pero perteneciente a otra comunidad autónoma, no escapa tampoco a esa influencia, aunque de manera mucho más limitada, fundamentalmente a través de la formación que reciben sus niños y jóvenes en la comunidad vecina, lo que implica aprender la lengua vasca de manera reglada. Por su parte, Castro ha recibido en las últimas décadas un ingente número de nuevos habitantes procedentes mayoritariamente de la margen izquierda del Nervión, en territorio vasco pero con baja euskaldunización. Todos estos factores han repercutido en el diferente empleo y presencia del español y del vasco en cada área. A efectos de esta investigación esa distribución de lenguas no será tenida en consideración, ${ }^{9}$ ya que responde a otras causas y no al ámbito de desarrollo (rural o urbano) de las lenguas. Nos centraremos, por

\footnotetext{
5 De acuerdo con Delgado Viñas (2018) y también con la información suministrada por el propio Ayuntamiento de Castro.

${ }^{6}$ Hasta esa fecha el INE registra cifras entre los 14191 de 1900 y los 11646 de 1950. Coincide el ascenso demográfico de la década de lo 90 con la mejora de las comunicaciones mediante la apertura de la autovía del Cantábrico, que ha reducido considerablemente el trayecto entre Bilbao y Castro. Se han aducido otros motivos que explican el movimiento migratorio: el precio más favorable de la vivienda (Delgado Viñas, 2018) y la amenaza terrorista en el País Vasco.

${ }^{7}$ Un 74,1\% de la población activa de 2017 frente al 19,8\% que se dedica a la construcción y un 4,7\% a la industria (Delgado Viñas, 2018).

8 Valle de Villaverde es un enclave de una comunidad autónoma, Cantabria, en otra, el País Vasco (Carnicer, 1995). Se trata de una situación no extraña pero tampoco habitual en la distribución territorial de España y también en la de otros países. En el caso del Valle de Villaverde, ocupa apenas 19,5 km² dentro de la comarca vizcaína de Las Encartaciones.

${ }^{9}$ Los usos lingüísticos de ambas zonas han sido tratados en estudios previos (Fernández Juncal, en prensa).
} 
lo tanto, en otros parámetros, los tipos de señales que predominan en los dos ámbitos (rural y urbano), que pueden ser extensibles a otras realidades de índole semejante. La determinación de la presencia y clasificación de textos nos ayudará a dilucidar los rasgos que definen uno y otro paisaje en líneas generales, y especialmente a caracterizar el paisaje rural, que, como hemos advertido, ha sido relegado en los estudios de esta naturaleza.

\section{METODOLOGÍA}

\section{Mapa 1. Situación de las dos áreas de estudio: urbano (Castro Urdiales) y rural (Valle de Villaverde y Trucíos-Turtioz). Elaboración propia a partir de mapa del IGN}

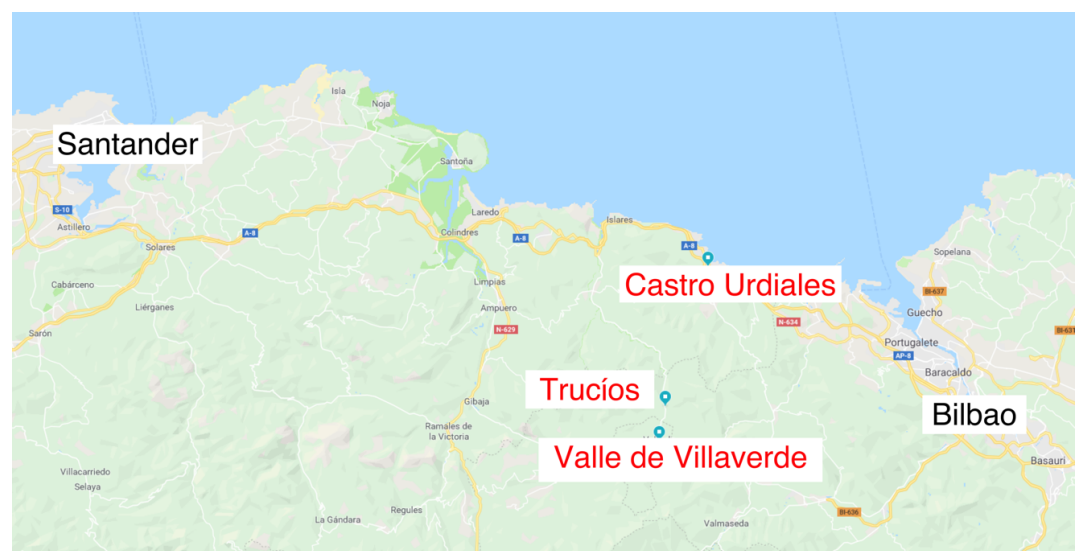

El trabajo de campo se llevó a cabo a lo largo del verano de 2017 en las capitales administrativas de los dos municipios rurales: La Matanza, de Valle de Villaverde, y La Iglesia, de Trucíos-Turtzioz, ambas colindantes (ver mapa 1). Es allí donde tienen sus sedes los organismos administrativos y se concentran las escasas infraestructuras de servicios de ambos municipios.

En lo que respecta al núcleo urbano de Castro, la recogida de datos se realizó también a lo largo del verano de 2018 en una zona acotada: el centro comercial de la ciudad, que coincide con el que era núcleo urbano antes de la expansión de mediados del siglo XX. Esta área está delimitada de manera inequívoca por el trazado de la antigua carretera nacional que antes bordeaba y ahora cruza la población. Allí tienen sus sedes los organismos administrativos más importantes y se concentran las infraestructuras de servicios comerciales y hosteleros.

La recogida de datos se llevó a cabo siguiendo dos postulados: el primero exigía que los signos que formaran el corpus poseyeran estatismo, duradero o precario. Esto ha provocado que no se incluyeran en el conjunto de señales elementos móviles o semimóviles, como textos en vehículos, ropa de personas, tatuajes, etc. (Pons, 2012). El segundo requisito venía dado por las limitaciones que padecían las entidades rurales en su paisaje: era necesario garantizar el principio de exhaustividad que fundamenta este tipo de investigaciones, sin establecer restricciones de grado de visualización, lo que hace incluir en el corpus todos los textos existentes aunque sean de pequeño formato o lo que Castillo y Sáez (2011) llaman signos parásitos (anuncios fijados en lugares no destinados a albergarlos). En resumen, se consignaron todos los signos (y no una representación) 
dentro de las áreas de estudio: el centro comercial (centro histórico simultáneamente) de Castro y la totalidad del territorio de las dos localidades rurales. ${ }^{10}$

La información lingüística y extralingüística fue convenientemente fotografiada y trasladada a una base de datos, en la cual se han registrado cuantos pormenores pudieran ser significativos. A partir de estos datos se han clasificado las señales de acuerdo con los parámetros que habitualmente se utilizan en los estudios sobre paisaje lingüístico: autoría, fijación, función, alcance, fecha y lengua de empleo.

En primer lugar, consideraremos su autoría. Se trata de un criterio de gran importancia, pero no exento de dificultades, como las señaladas por Pons (2012: 77-78):

¿Quién es el autor del texto? ¿La persona que lo ha ideado, quien lo ha impreso o quien lo está mostrando? [...] En efecto, la heteroglosia que es para estos teóricos connatural a los enunciados lingüísticos se plasma de forma manifiesta en buena parte de los signos públicos del paisaje lingüístico.

Tradicionalmente se habla de textos públicos u oficiales (top-down) o privados (bottom-up), aunque también podemos encontrar otros que son de carácter polifónico (textos mixtos institucionales con publicidad pseudoencubierta ${ }^{11}$ ), como vemos en a imagen 1 .

\section{Imagen 1. Convivencia de señalespúblicas y privadas en medio rural} (Trucíos/Turtzioz)

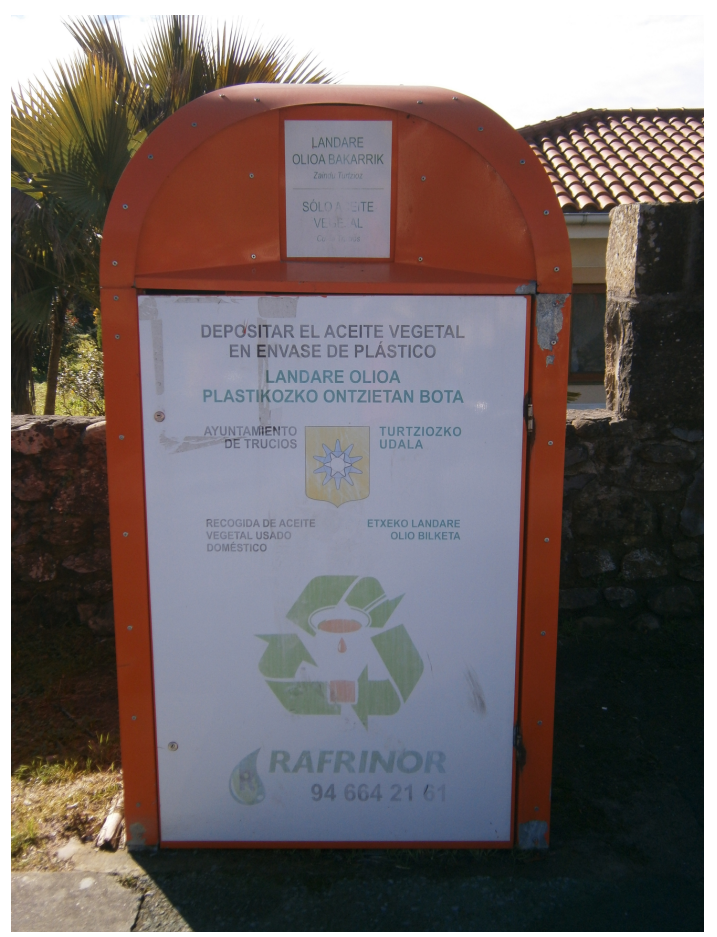

${ }^{10}$ Para los cálculos de densidad de textos lingüísticos empleamos como referencia la superficie total del terreno. Esta decisión tiene la limitación de que la mayor parte es ocupada por uso privado y no mide exactamente los metros cuadrados de fachada de acceso público, pero nos sirve de punto de partida para el contraste.

${ }^{11}$ Ocurre con algunos signos de autoría pública como señales, alcantarillas, etc. en los que se ha incluido publicidad de la empresa que los ha realizado o desarrollado. 
El segundo criterio de clasificación de las señales, muy relacionado con la autoría, es su alcance, es decir, el origen y extensión de los textos. Así, el alcance puede ser local, ampliado o global, dependiendo de si se han originado en el mismo lugar donde se muestran, si pertenecen al ámbito regional o nacional o si son signos de ámbito internacional.

En tercer lugar, hemos empleado como criterio de clasificación el grado de permanencia de los elementos del corpus, es decir, su fijación. Sabemos que no existe elemento del paisaje que no pueda alterarse o desaparecer, pero, a efectos de este parámetro, distinguimos los signos permanentes, que están fijados con voluntad de continuidad y lo que es caduco o efímero. Este factor tiene una relación de dependencia del soporte utilizado. Así, son más duraderos los elementos inscritos en material de una cierta solidez (metal, piedra) que aquellos que se sustentan en material más endeble, como el papel. No obstante, encontramos unidades con aparente fecha de caducidad, sin voluntad de permanecer, que se mantienen de manera más o menos deliberada, perdiendo su valor informativo y desempeñando entonces una función simbólica (Bourhis, 1992). ${ }^{12}$ Muy vinculado con este factor está un cuarto, la fecha de creación del signo, a veces difícil de establecer. En este caso se distinguen tres periodos: periodo $1^{\circ}$ (1975-2017), periodo $2^{\circ}(1930-1979)$ y periodo $3^{\circ}$ (anterior a 1930)..$^{13}$

En quinto lugar, tendremos en cuenta su finalidad. Para ello hemos tomado como referencia la propuesta de clasificación de Pons (2012), y la hemos adaptado al conjunto de escritos recabado para esta investigación. En total identificamos once funciones: señalización vial, marcas toponímicas, información institucional, textos publicitarios, avisos comerciales, otros avisos (anuncios particulares), textos nominales, textos conmemorativos, pintadas e incisiones, información turística y otros textos, que son de tipo misceláneo y donde se integran, por ejemplo, los de carácter político. En caso de confluencia de funciones, como ocurre en ocasiones, se selecciona la función primordial.

Finalmente hemos advertido que no nos servirá para nuestros intereses el empleo de una lengua u otra en el paisaje, sobre todo las lenguas afectadas en esta zona, vasco y español, porque la distribución de estas no viene dada por el carácter rural o urbano de las sintopías analizadas sino por razones idiosincrásicas de cada una de ellas (perfil y desarrollo histórico lingüísticos, aplicación de políticas lingüísticas, etc.). No obstante, sí tendremos en cuenta como sexto criterio de estudio el uso lingüístico general, la aparición de otras variedades, es decir, la diversidad y riqueza lingüísticas. A este respecto, aunque también puedan ser empleados en otra, se han catalogado dentro de su lengua original los nombres propios, antropónimos y topónimos, sobre todo, los nombres de persona. Esta decisión secunda la postura de Edelman (2009) de no minusvalorar este tipo de vocablos por el valor que tienen como ejemplificadores de los diferentes usos multilingües.

\section{ANÁLISIS DE DATOS}

El corpus resultante del trabajo de campo es una colección de 217 textos en las dos localidades rurales y 1299 en la ciudad. ${ }^{14}$ Solo este dato ya es muy indicativo de las diferencias entre los dos entornos, pero debemos considerarlo en relación con las

\footnotetext{
${ }^{12}$ Es lo que ocurre con carteles de obras institucionales, que en muchos casos han perdido vigencia pero mantienen su valor de intervención institucional.

${ }^{13}$ La segmentación que se hizo en la investigación relativa a estos paisajes lingüísticos vino dada por la relación de estos periodos históricos con diferentes actuaciones institucionales hacia las lenguas de estudio (español y vasco).

${ }^{14}$ El número de signos analizados no siempre va en consonancia con las dimensiones de la población estudiada sino, más bien, con los objetivos de cada investigación. En cualquier caso, en los estudios de PL se observan muchas fluctuaciones en la cifra de textos de cada corpus.
} 
dimensiones que ocupan y con los usuarios del espacio. A partir de las herramientas proporcionadas por el Catastro Virtual, se han realizado mediciones de los terrenos, que adjudican 12,7 hectáreas para el centro de Castro y 23,4 hectáreas para la suma de los centros de la Matanza y la Iglesia, capitales respectivas de Valle de Villaverde y Trucíos/Turtzioz. Por lo tanto, la densidad de señales es de 102,28 signos/ha en medio urbano y de 9,27 signos/ha en medio rural.

La confrontación de cifras es evidente. La riqueza y concentración de señales lingüísticas en ambos entornos es muy desigual. En la ciudad se produce una estimulación más de 10 veces superior a la que se recibe en los dos pueblos. Esta disparidad cuantitativa tendrá consecuencias directas en la caracterización del paisaje lingüístico. En la tabla 1 se desglosa la distribución de los textos de acuerdo con los criterios detallados en el apartado $3:{ }^{15}$

Tabla 1. Caracterización de los corpus de acuerdo con los criterios autoría, fijación, fecha y alcance

\begin{tabular}{|c|c|c|c|c|c|}
\hline & & \multicolumn{2}{|c|}{ URBANO } & \multicolumn{2}{|c|}{ RURAL } \\
\hline & & $\mathrm{N}^{\circ}$ & $\%$ & $\mathrm{~N}^{\circ}$ & $\%$ \\
\hline \multirow[t]{2}{*}{ AUTORÍA } & Pública & 149 & 11,47 & 118 & 54,38 \\
\hline & Privado & 1150 & 88,53 & 99 & 45,62 \\
\hline \multirow[t]{3}{*}{ ALCANCE } & Local & 1047 & 80,6 & 122 & 56,22 \\
\hline & Ampliado & 206 & 15,86 & 91 & 41,94 \\
\hline & Global & 46 & 3,54 & 4 & 1,84 \\
\hline \multirow[t]{3}{*}{ FECHA } & Periodo $1^{\circ}$ & 1171 & 90,15 & 200 & 92,16 \\
\hline & Periodo $2^{\circ}$ & 127 & 9,77 & 14 & 6,46 \\
\hline & Periodo $3^{\circ}$ & 1 & 0,08 & 3 & 1,38 \\
\hline \multirow[t]{3}{*}{ FIJACIÓN } & Permanente & 626 & 48,19 & 150 & 69,12 \\
\hline & Caduco & 673 & 51,81 & 67 & 30,88 \\
\hline & TOTAL & 1299 & & 217 & \\
\hline
\end{tabular}

Si aplicamos la prueba de ji-cuadrado $\left(\chi^{2}\right)$, los resultados avalan la pertinencia de los criterios seleccionados. En todos los casos el grado de probabilidad es significativo al nivel $\mathrm{p}<0,001$, especialmente revelador. Con ello se refuta la hipótesis nula y se confirma la correlación entre los factores analizados y su empleo y distribución en medio rural y urbano.

Los parámetros más discriminantes son los dos primeros, que como decíamos, están relacionados entre sí. En lo que concierne a la autoría, en el entorno urbano predomina la actividad industrial y de servicios, principal fuente de textos de autoría privada, que representa una mayoría sobresaliente respecto a los textos de origen

\footnotetext{
${ }^{15}$ Utilizaremos a lo largo de este estudio como referencia estadística básica la frecuencia, un dato sencillo pero muy eficaz para este tipo de análisis. La hallamos considerando como universo los datos globales de textos recogidos, entendiendo estos como unidad-signo y que se han detallado al comienzo de este apartado. Además de las frecuencias relativas, se aportarán también las absolutas. Completaremos estas estadísticas con pruebas de ji-cuadrado, que nos ayudarán a valorar la significación de los parámetros empleados.
} 
institucional. Esta situación concuerda con otras investigaciones, como la de Ariolfo (2017) en ámbito urbano (Génova), donde apenas llegan a un 9\% los textos de este carácter o la de Franco-Rodríguez en Miami-Dade (2007), que registra un 6\% de textos públicos.

Estado diferente es el que presenta el entorno rural: más de la mitad de los textos emanan de una institución de carácter público, sea del propio municipio, de la comunidad autónoma o nacional, incluso europea. Creemos que, si bien las diversas administraciones (local, regional, nacional) hacen visible su presencia en poblaciones pequeñas (vemos un ejemplo en la imagen 2), las causas de su ubicuidad deben achacarse más bien a la reducción de los signos privados, cuya ausencia se debe a una estructura económica menos dependiente del sector servicios, que no propicia la creación y el empleo de textos por parte de particulares. Estas desemejanzas en la organización socioeconómica son en su mayor parte la razón que explica las disparidades en el paisaje lingüístico de los dos ámbitos.

\section{Imagen 2. Presencia institucional en medio rural (Valle de Villaverde)}

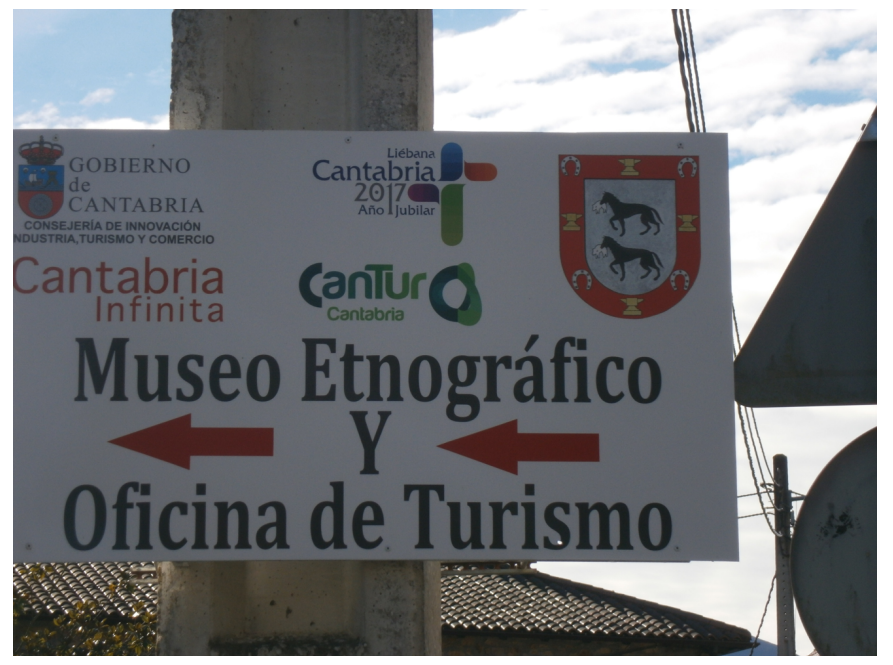

El alcance de los textos es primordialmente local en ambos casos, pero con una diferencia muy significativa a favor del medio urbano, que manifiesta mayor autonomía en la generación de su propio paisaje, en gran parte por no depender de la iniciativa pública. En contraste, el medio rural genera menos textos propios porque sencillamente, debido a su estructura económica, no los precisa y, como contrapartida, asimila elementos provenientes de otros lugares en el dominio territorial inmediato (provincia, región). Por último, en números absolutos, la ciudad es mayor receptora de signos globales, pero porcentualmente no existe una brecha entre los dos ámbitos. En general, los signos de carácter internacional tienen una presencia limitada pero constante, más si consideramos la complejidad de su expansión.

Son datos más igualados los que hallamos referidos a la fecha y fijación de los textos, factores que poseen una estrecha vinculación. La fijación es más permanente en medio rural, dato que podemos atribuir en parte al empleo simbólico de algunas señales por parte de instituciones públicas: ocurre que muchas iniciativas de carácter temporal (inversiones, obras, etc.), están en un soporte duradero, lo que alarga su vida y, como señalamos en el apartado 3, hace que adquieran una función emblemática de presencia institucional más prolongada. No olvidemos además que los cambios de elementos están muy ligados a la necesaria reposición que implica la actividad hostelera y comercial y que esta se produce con más asiduidad en medio urbano. 
Sin embargo, en líneas generales conviven en el mismo espacio soportes de diferente índole, lo que hace que el paisaje se renueve constantemente con aquellos que son más endebles y tienen vida más corta, pero también con otros en apariencia más perecederos pero que se modifican en paralelo al dinamismo del mundo empresarial. Esta regeneración de la mayor parte de los elementos del corpus se confirma con las cifras relativas a sus fechas de creación: las señales se concentran en las últimas décadas y apenas unas pocas pertenecen a periodos anteriores ${ }^{16}$ (ver imagen 3 ).

\section{/ Turtzioz)}

\section{Imagen 3. Convivencia de signos de diferentes épocas en medio rural (Trucíos}

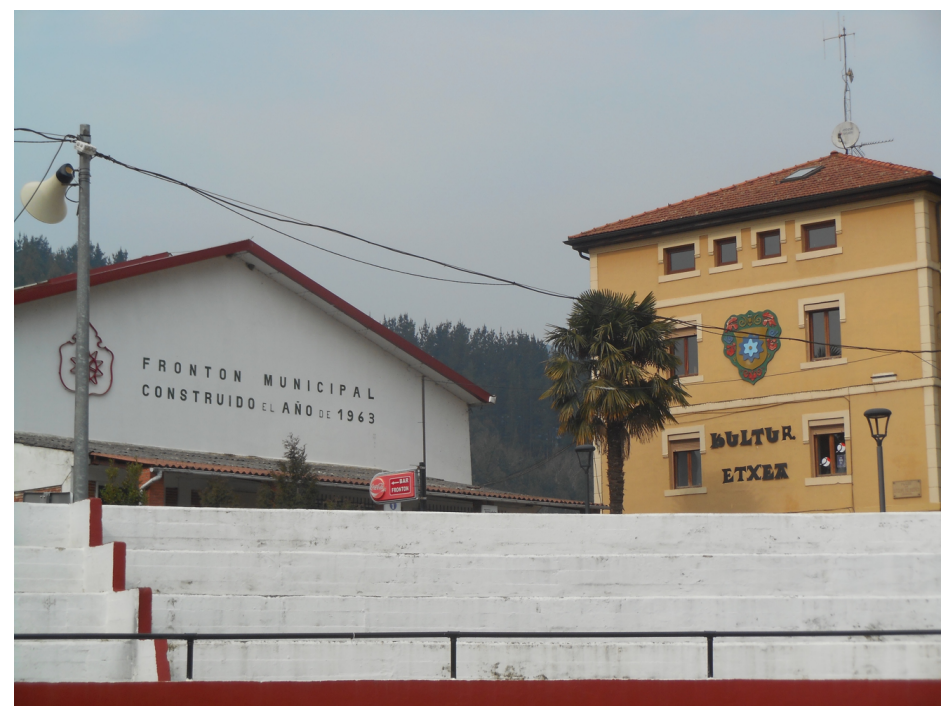

Consideraremos a continuación el quinto parámetro, la finalidad que los textos cumplen en su entorno (ver tabla 2).

\section{Tabla 2. Caracterización de los corpus de acuerdo con el criterio función}

\begin{tabular}{|l|c|c|c|c|}
\hline & \multicolumn{2}{|c|}{ URBANO } & \multicolumn{2}{c|}{ RURAL } \\
\hline FUNCIÓN & $\mathbf{N}^{\mathbf{0}}$ & $\mathbf{\%}$ & $\mathbf{N}^{\mathbf{0}}$ & $\mathbf{\%}$ \\
\hline Señal vial & 22 & $\mathbf{1 , 7}$ & 30 & $\mathbf{1 3 , 8}$ \\
\hline Toponimia & 31 & $\mathbf{2 , 4}$ & 8 & $\mathbf{3 , 7}$ \\
\hline Info. institucional & 61 & $\mathbf{4 , 7}$ & 53 & $\mathbf{2 4 , 5}$ \\
\hline Publicidad & 119 & $\mathbf{9 , 2}$ & 22 & $\mathbf{1 0 , 1}$ \\
\hline Avisos comerciales & 370 & $\mathbf{2 8 , 5}$ & 25 & $\mathbf{1 1 , 5}$ \\
\hline Otros avisos & 21 & $\mathbf{1 , 6}$ & 4 & $\mathbf{1 , 8}$ \\
\hline Nominales & 449 & $\mathbf{3 4 , 6}$ & 55 & $\mathbf{2 5 , 4}$ \\
\hline Conmemoración & 8 & $\mathbf{0 , 6}$ & 7 & $\mathbf{3 , 2}$ \\
\hline Pintadas e incisiones & 168 & $\mathbf{1 2 , 9}$ & 3 & $\mathbf{1 , 4}$ \\
\hline Inf turística & 13 & $\mathbf{1}$ & 6 & $\mathbf{2 , 8}$ \\
\hline Otros & 37 & $\mathbf{2 , 8}$ & 4 & $\mathbf{1 , 8}$ \\
\hline
\end{tabular}

Como en la tabla anterior, de la aplicación de la prueba de ji-cuadrado $\left(\chi^{2}\right)$ resulta la pertinencia de la variable función para explicar el empleo de los diferentes signos en el paisaje con una significatividad de $p<0,001$.

\footnotetext{
${ }^{16}$ Esta información es muy útil para análisis de tipo lingüístico en el caso de implantación de políticas lingüísticas con fecha determinada.
} 
La tabla 2 nos permite ver, en primer lugar, los puntos de encuentro de ambos paisajes. Son funciones menores los datos toponímicos, los anuncios personales (se han catalogado como otros avisos), los textos de carácter conmemorativo, la información turística y otras funciones, como la comunicación política. Apenas si llama la atención que la información turística sea superior en los pueblos que en la ciudad, dato atribuible al cambio económico que los primeros están experimentando a favor de un sector que deben potenciar y donde la información de este tipo resulta necesaria frente a lugares que poseen una trayectoria en esa parcela.

Más esclarecedoras son las cifras acerca de las funciones principales, donde se producen las mayores discrepancias entre ambos paisajes. Los dos entornos comparten la primera posición en las funciones del paisaje: nombrar espacios, pero con una frecuencia más elevada para el caso de la ciudad, donde sencillamente existen más elementos de denominación. Resulta más llamativa la divergencia en la transmisión de información institucional, muy relevante en medio rural, en consonancia con los datos relativos a la autoría pública de los textos, y mucho más limitada en medio urbano, donde predomina, como vimos, la iniciativa privada sobre la oficial. Se reafirma, por lo tanto, uno de los rasgos diferenciadores entre ambos dominios.

Tiene también distinto peso la presencia de señalización vial, de origen público, más necesaria en un territorio que cuenta con vías de comunicación interpoblacional, como es el rural. También podemos aducir las mismas causas generales para justificar el mayor empleo de avisos comerciales en la ciudad, donde el sector terciario posee mayor relieve y trascendencia.

Finalmente, se observa un contraste notorio en la existencia de pintadas e incisiones, fenómeno casi ausente en medio rural, asociado en su origen y desarrollo al contexto urbano (ver imagen 4), como ratifican Vigara y Reyes (1997):

En su ámbito, por definición urbano, [la pintada] nace acorralada por normas, reglas, códigos, pautas y comportamientos que le son ajenos, pertenecientes a una dimensión normalizada de las relaciones sociales, al margen de las cuales se establece. $\mathrm{Y}$ es, en este sentido, como se ha dicho siempre, marginal, voluntariamente marginal y, por ello, socialmente repudiada e ilegal. Pero no tanto por la procedencia sociocultural, muy diversa, de sus autores (en contra de lo que tantas veces se ha dicho también), cuanto por cómo éstos infringen las más elementales normas de convivencia haciendo uso (que no se les ha concedido) de la palabra (que nadie les ha pedido) y de un espacio que no sólo no les pertenece, sino que les está expresamente prohibido.

\section{Imagen 4. Proliferación de pintadas en medio urbano (Castro Urdiales)}

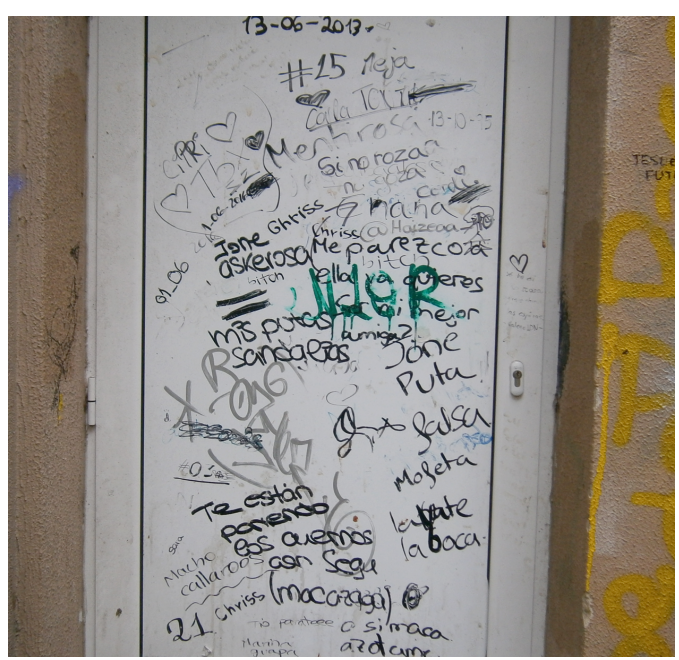


Siguiendo la nomenclatura de estos autores, en nuestro corpus se encuentran tanto pintadas (mensajes centrados en la comunicación) como graffiti (mensajes centrados en la forma). Estos últimos concentran, como veremos a continuación, todos los textos de lengua no identificada.

Como avanzábamos, para finalizar el análisis nos detendremos brevemente en la variación lingüística de ambos paisajes. Sin embargo, al contrario de lo que suele ocurrir en los estudios de esta naturaleza, no pondremos el foco en la distribución cuantitativa de lenguas que conviven en un territorio, que viene dada por otros factores ajenos a su carácter rural o urbano, sino en su diversidad, la variedad lingüística del PL. Vemos el desglose en la tabla 3:

Tabla 3. Caracterización de los corpus de acuerdo con el criterio lengua

\begin{tabular}{|c|c|c|c|}
\hline MEDIO & TEXTOS & LENGUAS (no) & $\%$ \\
\hline \multirow[t]{10}{*}{ URBANO } & Textos monolingües & & \\
\hline & & español & 66,4 \\
\hline & & Inglés & 5,1 \\
\hline & & Vasco & 0,9 \\
\hline & & Otras lenguas (7 lenguas) & 1,6 \\
\hline & Textos bilingües y mixtos & & \\
\hline & & Español-inglés & 7,1 \\
\hline & & Español-vasco & 6,5 \\
\hline & & $\begin{array}{l}\text { Español-otras lenguas } \\
\text { / otras combinaciones ( } 14 \text { lenguas) }\end{array}$ & 3,7 \\
\hline & Desconocida & & 8,7 \\
\hline \multirow[t]{9}{*}{ RURAL $^{17}$} & Textos monolingües & & \\
\hline & & español & 61,3 \\
\hline & & Inglés & 2,8 \\
\hline & & Vasco & 7,3 \\
\hline & Textos bilingües y mixtos & & \\
\hline & & Español-inglés & 2,8 \\
\hline & & Español-vasco & 25,8 \\
\hline & & $\begin{array}{l}\text { Español-otras lenguas } \\
\text { / otras combinaciones }\end{array}$ & - \\
\hline & Desconocida & & - \\
\hline
\end{tabular}

El español es la lengua más utilizada en todos los contextos, de manera independiente o con elementos insertos de otras lenguas (83,2 en medio urbano y 89,9 en medio rural). El inglés, lengua de comunicación y extensión internacional, tiene presencia constatada en ambos contextos, pero de manera más acentuada en el urbano, donde las circunstancias propician este uso por la intensidad de la actividad comercial, uno de cuyos reclamos es el empleo de formas cargadas de connotaciones positivas. Esta presencia confirma su papel como canal de la globalización: su función no es tanto informativa como simbólica, activando valores como la internacionalización, la modernidad, el éxito, la sofisticación, etc. (Cenoz y Gorter, 2009).

En lo que se refiere a otras lenguas, el dato más significativo es la escasez de ellas en medio rural, donde se encuentra solo el vasco, la lengua cooficial o vecina, como única representante. En medio urbano hay otras 7 lenguas (francés, italiano, alemán, catalán,

${ }^{17}$ En la interpretación del cuadro téngase en cuenta que se presenta la suma de las dos localidades rurales, Valle de Villaverde (Cantabria) y Trucíos/Turtzioz (País Vasco), y que el peso primordial de los porcentajes del vasco recaen, como es natural, en la segunda. 
ruso, sueco y turco) que aparecen de forma autónoma en textos monolingües y otras 7 (japonés, chino, neerlandés, portugués, árabe, latín y rumano), que, además de las anteriores, conviven con el español o con otras lenguas en textos mixtos. Como hemos apuntado, la diversidad, la heterogeneidad lingüística tienen en la ciudad el mejor campo de cultivo, apoyadas por una población de estructura más compleja, que favorece las condiciones para desarrollar entornos multilingües y multiculturales (ver imagen 5).

\section{Imagen 5. Texto multilingüe en medio urbano}

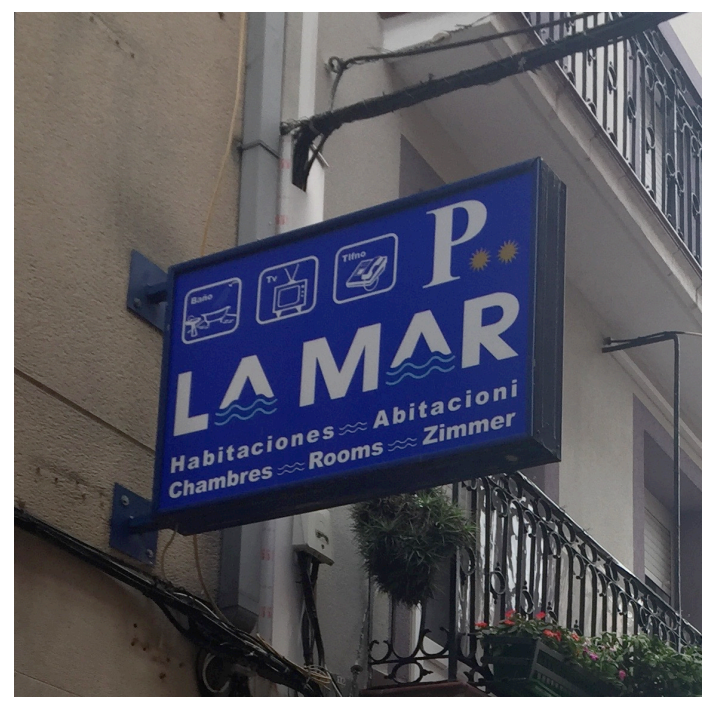

\section{CONCLUSIONES}

En este estudio hemos llevado a cabo una comparación del paisaje lingüístico de dos áreas, una de carácter urbano y otra de carácter rural. Como advertíamos en la introducción, el PL urbano, por su propia naturaleza, por su multiplicidad demográfica y socioeconómica, ha constituido el centro de atención de esta clase de estudios, mientras que el PL rural no ha disfrutado de ese interés, a pesar de que se ha repetido en ocasiones (Gorter, 2006) el provecho y atractivo que tendría diversificar el área de observación. Así, apenas contamos con algunas investigaciones en este medio.

Basándonos en investigaciones previas en medio rural y urbano, observamos la cuantificación de diversos parámetros que puede servir para la caracterización de los corpus resultantes. Los puntos de contacto de ambos entornos son la fecha y fijación de las señales, donde no se aprecian diferencias significativas: en ambos casos la mayoría de los textos son de fecha reciente y conviven de manera equilibrada los que tienen carácter más permanente junto con los perecederos.

Sin embargo, en otros factores se observan disimilitudes relevantes. La autoría de las señales tiene signo diferente: mayoritariamente privada para la ciudad y pública para los pueblos. Este dato tiene también secuelas en su alcance; el medio urbano genera más elementos propios de proyección interna, mientras que el medio rural incorpora en mayor medida componentes externos y su paisaje es subsidiario y compartido con el territorio administrativo más próximo (provincia, región).

Hay disparidades también significativas en lo que concierne a la función de los signos. El cometido principal en ambos casos en el de nombrar lugares, establecer marcas comerciales para la identificación de establecimientos. La dinámica público-privado que hemos mencionado explica también la importancia de la información institucional (lo que incluye la señalización vial) como segundo desempeño de los textos rurales, mientras que 
su presencia es mucho menor en la ciudad. A la luz de esa misma oposición podemos interpretar el diferente peso de los avisos comerciales a favor del contexto urbano y la presencia en este mismo entorno de un fenómeno prácticamente ausente de los pueblos: graffiti y pintadas.

Finalmente, si consideramos la diversidad lingüística presente en ambos medios, esta es mucho más notoria en la ciudad, donde conviven personas de diferentes orígenes, donde se ponen en marcha estrategias de persuasión que incluyen innovaciones de todo tipo, también lingüísticas. En palabras de Claus et al. (apud Gorter, 2006), «[signs] serve multiple functions beyond indexing, including marketing, advertising, way-finding, providing information, building image, educating, and creating a visually stimulating retail environment».

Ratificamos a partir de lo expuesto, el interés del medio rural en los estudios de paisaje lingüístico y lo confirmamos por diversas razones: en primer lugar, porque presenta una tipología diferente que viene dada por la limitación de actores y la base económica en la que se sustenta. De esa forma se restringen y distinguen el número de signos y su tipología, más nominativa e informativa, menos persuasiva y comercial. En segundo lugar y como consecuencia de lo anterior, se puede observar con especial perspectiva el papel destacado de las instituciones en el espacio público, asunto que puede presentar un interés particular en los múltiples casos en que se están desarrollando acciones de política lingüística, que pueden a su vez provocar efectos en el área privada. Como indica Pons (2012: 66),

a menudo los signos creados por las instituciones otorgan una visibilidad en el escenario a un grupo que en cambio no la tiene en el colectivo; desarrollan una planificación participativa del paisaje tratando de ejemplarizar a partir de los signos que crean y, a partir de ahí, se pueden producir descoordinaciones que son muy reveladoras sobre las creencias, convicciones y repartos funcionales con que los hablantes usan los idiomas.

Finalmente, esta investigación pone de manifiesto el rendimiento y la eficacia de la aplicación del concepto y metodología sobre paisajes lingüísticos en la descripción de diferentes espacios públicos (Ariolfo, 2017) como reflejo de procesos de globalización y de cambios sustanciales en la demografía de los agrupamientos humanos y las representaciones sociales. Sirva, por lo tanto, este trabajo como paso adelante en la propia configuración de la subdisciplina, que afronta otros retos conceptuales y metodológicos: crear pautas de comparabilidad entre investigaciones, ampliar el origen y la tipología de las fuentes y profundizar en su necesaria perspectiva multidisciplinar.

\section{REFERENCIAS BIBLIOGRÁFICAS}

Ariolfo, Rosana. 2017. «Visibilidad y percepción del español en el paisaje lingüístico genovés». Lingue e Linguaggi, 21: 7-25.

Backhaus, Peter. 2006. Signs of Multilingualism in Tokyo: A Linguistic Landscape Approach. Clevedon: Multilingual Matters.

Baigorri, Artemio. 1995. «De lo rural a lo urbano. Hipótesis sobre las dificultades de mantener la separación epistemológica entre Sociología Rural y Sociología Urbana en el marco del actual proceso de urbanización global». https://www.eweb.unex.es/eweb/sociolog/BAIGORRI/papers/rurbano.pdf.

Barni, Monica y Carla Bagna. 2015. «The critical turn in LL. New methodologies and new items in LL». Linguistic Landscape, vol 1, 1/2: 6-18.

Ben-Rafael, Eliezer y Miriam Ben-Rafael. 2015. «Linguistic landscapes in an era of multiple globalizations». Linguistic Landscape, 1, 1/2: 19-37. 
Blackwood, Robert. 2015. «LL explorations and methodological Challenges. Analysing France's regional languages». Linguistic Landscape, 1, 1/2:38-53.

Blommaert, J. 2010. A sociolinguistics of globalization. Cambridge: Cambridge University Press.

Bourhis, R. 1992. La langue d'affichage publique et commerciale au Québec. Plan de recherche pour l'élaboration d'une loi linguistique. Quebec: Conseil de la langue française.

Burr, V. 2003. Social constructionism. London: Routledge.

Carnicer, Ramón. 1995. Viaje a los enclaves españoles. Sabadell: Ausa.

Castillo Lluch, Mónica y Daniel Sáez Rivera. 2011. «Introducción al paisaje lingüístico de Madrid». Lengua y Migración, 3:1: 73-88.

Cenoz, J. y D. Gorter. 2008. «El estudio del paisaje lingüístico». Hizkunea, 22. www.euskara.euskadi.net/r59-

bpeduki/es/contenidos/informacion/artik22_1_cenoz_08_03/es_cenoz/artik22_1_c enoz_08_03.html.

—. 2009. «Language economy and linguistic landscape». En Linguistic Landscape expanding the scenery, eds. E. Shohamy, D. Gorter. London: Routledge, pp. 5569.

Delgado Viñas, C. 2018. «Los procesos de metropolitanización dispersa: Castro Urdiales (Cantabria) en la región urbana de Bilbao». Boletín de la Asociación de Geógrafos Españoles, 78: 474-517.

Edelman, Loulou. 2009. "What's in a name? Classification of Proper Names by Language». En Linguistic Landscape - expanding the scenery, eds. E. Shohamy y D. Gorter. London: Routledge, 141-154.

Fernández Juncal, Carmen. en prensa. «El paisaje lingüístico de un área rural: frontera, transición y contacto». Bulletin of Hispanic Studies.

Franco-Rodríguez, J.M. 2007. «El español en el Condado de Miami-Dade desde su paisaje lingüístico». Lingüistica en red, 5: 1-29.

—. 2008. «El paisaje lingüístico del condado de Los Ángeles y del condado de MiamiDade: propuesta metodológica». Círculo de Lingüística aplicada a la Comunicación, 35: 3-43.

Gorter, D. 2006. «Further Possibilities for Linguistic Landscape Research». En Linguistic Landscape: a new approach to multilingualism, ed. D. Gorter. Clevedon: Multilingual Matters, 81-89.

—. 2012. «Forward». En Linguistic Landscapes, Multilingualism and Social Change, eds. C. Hélot, M. Barni, R. Janssens y C. Bagna. Frankfurt: Peter Lang, 9-12.

—. 2013. «Linguistic landscapes in a multilingual world». Annual Review of Applied Linguistics, 33: 190-212.

Gorter, D. y J. Cenoz. 2015. «Translanguaging and linguistic landscapes». Linguistic Landscape, 1, 1/2: 54-74.

Guerra Salas, L. (2018), «El paisaje lingüístico desde la lingüística perceptiva». Lingue e linguaggi, 25: 125-143.

Huebner, T. 2016. «Linguistic landscape: history, trajectory and pedagogy». MANUSYA, 22: $1-11$.

Juffermans, K. y J. Coppoolse. 2012. «How literate, low-literate and non-literate readers read the linguistic landscape in a Gambian village». En Linguistic landscapes, multilingualism and social change, eds. C. Hélot, M. Barni, R. Janssens y C. Bagna. Frankfurt: Peter Lang, 233-247.

Landry, R. y R. Bourhis. 1997. «Linguistic landscape and ethnolinguistic vitality: an empirical study». Journal of Language and Social Psychology, 16 (23): 23-49. 
López Serena, Araceli. 2008. «El sesgo escriptista en la historia de la reflexión sobre el lenguaje y en la ciencia lingüística contemporánea: la escritura como instrumento conceptual y filtro analítico falaz». $\operatorname{Lyn} X, 7: 135-153$.

de Luna Villalón, Eugenia. 2013. «El uso del español en el paisaje lingüístico de una pequeña ciudad canadiense». Estudios de Lingüística Aplicada, 58: 9-27.

Muñoz Carrobles, D. (2010), «Breve itinerario por el paisaje lingüístico de Madrid». Revista de estudios sobre la ciudad como espacio plural, 2, 2: 103-109.

Pavlenko, A. 2010. «Linguistic landscape of Kyiv, Ukraine: A diachronic study». En Linguistic landscape in the city, eds. E. Shohamy, E. Ben-Rafael y M. Barni. Clevedon: Multilingual Matters, 133-150.

Pons Rodríguez, Lola. 2012. El paisaje lingüístico de Sevilla. Lenguas y variedades en el escenario urbano hispalense. Sevilla: Diputación de Sevilla.

- 2014. «El paisaje lingüístico de la frontera luso-española: multilingüismo e identidad». En Investigaciones sobre la enseñanza del español y su cultura en contextos de inmigración, ed. E. Bravo-García. Sevilla-Helsinki: Universidad de Sevilla, Helsingin Yliopisto, 70-93.

Segrelles Serrano, J.A. 2011. «Características básicas del medio rural». https://rua.ua.es/dspace/bitstream/10045/19094/1/Microsoft\%20Word\%20\%20TEMAS_3_4.pdf

Shohamy, E. y D. Gurter. 2009. «Introduction». En Linguistic Landscape: Expanding the scenery, eds. E. Shohamy y D. Gurter. New York: Routledge, 1-11.

Spolsky, B. 2009. «Prolegomena to a sociolinguistic theory of public signage». En Linguistic landscape: Expanding the scenery, eds. Elana Shohamy y Durk Gorter. New York: Routledge, 25-40.

Vigara Tauste, A.M. y P. Reyes Sánchez. (1997). «Graffiti y pintadas en Madrid: arte, lenguaje, comunicación». Espéculo, 4.

http://webs.ucm.es/info/especulo/numero4/graffiti.htm 